\title{
Application of Non-additive Measures and Integrals for Analysis of the Importance of Party Positions for Voting
}

\author{
Alexander Lepskiy ${ }^{a}$ and Vladimir Smolev ${ }^{b}$ \\ ${ }^{a}$ Department of Mathematics, Faculty of Economic Sciences, \\ Higher School of Economics, 20 Myasnitskaya Ulitsa, Moscow, 101000, Russia, alex.lepskiy@gmail.com \\ ${ }^{b}$ Yandex LLC, 16 Ulitsa Lva Tolstogo, Moscow, 119021, Russia, smolev_vladimir@mail.ru
}

\begin{abstract}
The paper shows the possibility of applying the tool of non-additive measures and the belief functions theory to solving a number of problems of significance analysis and conflict of the political party positions. The study was performed on a database of online polls of parties in Germany before the elections to the Bundestag in 2013 and the results of these elections. The possibility of finding the most significant groups of issues for voting, evaluating the political heterogeneity of society, assessing the importance of the positions of individual parties for voting, assessing the conflict of the party positions on important issues is shown.
\end{abstract}

Keywords: Non-additive measures, Choquet integral, Belief functions, Conflict measure, Political science analysis.

\section{Introduction}

Party-list proportional representation system is the most popular voting system in parliamentary elections. Online surveys among parties are conducted in some countries. Political parties formulate in these polls their point of view on some issues of relevance to voters before parliamentary elections. For example, such a service is well developed in Germany [31]. The data of such services and their comparison with the results of subsequent elections are analyzed by both political scientists and data analysts [29]. For example, the following questions are of interest:

1. Are the political parties positions on topical issues of public life meaningful for voting in parliament?

2. How to identify groups of significant questions?

3. How to assess the political heterogeneity of society?
4. How to assess the consistency or conflict political party positions on important issues?

All these issues are widely discussed in the political science literature. For example, the consistency of voting between factions or within factions is analyzed in $[2,14]$. There are various (primarily game-theoretic) models for the formation and functioning of factions and coalitions $[16,19,22,23]$. The ability to create coalitions is often considered when analyzing the stability of the coalition [1].

The article presents the results of another approach. The above questions are set-functional character in terms of data analysis. Significance should not be considered for individual issues only, but on subsets of questions, since the 'synergistic' effect for the significance of certain groups of questions is possible. Therefore, it is necessary to use the appropriate tools to answer the questions posed. The tool of non-additive measures [11] and some methods of the belief functions theory $[7,25]$ can be such a tool, in our opinion.

The article presents the results of such a study on the example of online polls of parties in Germany before the elections to the Bundestag in 2013 [30]. The study reviewed and solved the problem of finding the most significant groups of issues for voting, the problem of assessing the influence of party positions on the voting result, the problem of assessing the conflicting political party positions on significant issues, the problem of evaluating the political heterogeneity of society.

\section{Preliminaries about Non-additive Measures, Integrals and the Belief Functions Theory}

Let $X=\left\{x_{1}, \ldots, x_{n}\right\}$ be some finite set (for example, set of all alternatives, criteria, features, etc.), $2^{X}$ be the powerset of $X$. Then the set function $\mu: 2^{X} \rightarrow$ $[0,1]$ is called the normalized non-additive measure on $X$ [11] (monotone measure, fuzzy measure, capacity) 
if it satisfies the conditions:

1. $\mu(\emptyset)=0, \mu(X)=1$ (grounded and normalized);

2. $\mu(A) \leq \mu(B)$, if $A \subseteq B, A, B \in 2^{X}$ (monotone).

The value of $\mu(A)$ characterizes the importance of the set of features (criteria, alternatives, etc.) $A$ for the problem being solved. In particular, the measure $\mu$ can be additive.

The discrete Choquet integral from the nonnegative function $f: X \rightarrow \mathbb{R}_{+}$by a non-additive measure $\mu$ on $X$ is calculated by the formula [5]

$$
C_{\mu}(f)=\sum_{i=1}^{n}\left(f\left(x_{\sigma(i)}\right)-f\left(x_{\sigma(i-1)}\right)\right) \mu\left(A_{\sigma(i)}\right),
$$

where $\sigma(i)$ is such an index ordering $\{1, \ldots, n\}$ that $0=f\left(x_{\sigma(0)}\right) \leq f\left(x_{\sigma(1)}\right) \leq \ldots \leq f\left(x_{\sigma(n)}\right), A_{\sigma(i)}=$ $\left\{x_{\sigma(i)}, \ldots, x_{\sigma(n)}\right\}, i=1, \ldots, n$. The Choquet integral, as an aggregating operator, finds numerous applications in pattern recognition problems $[12,15]$ and information fusion: multi-criteria decision making $[10,11]$, feature extraction [13], data fusion in multisensor systems [9, 28], etc.

The Choquet discrete integral is convenient to present in a number of problems in terms of the Möbius transformation $m^{\mu}: 2^{X} \rightarrow \mathbb{R}$ from non-additive measure $\mu$ :

$$
m^{\mu}(A)=\sum_{B \subseteq A}(-1)^{|A \backslash B|} \mu(B), \quad \mu(A)=\sum_{B \subseteq A} m^{\mu}(B)
$$

$A \in 2^{X}$. The value $m^{\mu}(A)$ characterizes the importance of the set $A$, regardless of its connection with its other subsets. The Choquet integral will have the following form in terms of the Möbius transformation [4]

$$
C_{\mu}(f)=\sum_{A \subseteq X} m^{\mu}(A) \min _{x \in A} f(x)
$$

If $m^{\mu}(A) \geq 0$ for all $A \in 2^{X}$, then non-additive measure $\mu$ is called a belief function. The dual measure is called plausibility function, and is defined by $\bar{\mu}(A)=1-\mu(\neg A), A \in 2^{X}$. Belief functions and plausibility functions are one of the main objects of the study of the belief functions theory (theory of evidence, the Dempster-Shafer theory) [7, 25].

The belief functions theory assumes that the mass function (also called basic probability assignment) $m$ : $2^{X} \rightarrow[0,1]$ is initially defined or statistically estimated. The belief function $\mathrm{Bel}$ and plausibility function $\mathrm{Pl}$ are then calculated by the formulas
$\operatorname{Bel}(A)=\sum_{B \subseteq A} m(B), \quad P l(A)=\sum_{B \cap A \neq \emptyset} m(B), \quad A \in 2^{X}$.

The contribution of individual alternatives to the overall importance of subsets of alternatives can be estimated using Shapley values [26], which are widely used in the theory of coalition games:

$$
v_{i}=\sum_{A \subseteq X, x_{i} \in A} \frac{(n-|A|) !(|A|-1) !}{n !} \Delta_{\left\{x_{i}\right\}} \operatorname{Bel}(A) .
$$

where $\Delta_{\left\{x_{i}\right\}} \operatorname{Bel}(A)=\operatorname{Bel}(A)-\operatorname{Bel}\left(A \backslash\left\{x_{i}\right\}\right)$. The vector $\mathbf{v}=\left(v_{i}\right)_{i=1}^{n}$ is called the Shapley vector, $\sum_{i=1}^{n} v_{i}=\operatorname{Bel}(X)=1$. It is known [27] that Shapley value of belief function coincides with so called pignistic transform

$$
v_{i}=\sum_{x_{i} \in D} \frac{m(D)}{|D|}
$$

The set $A \in 2^{X}$ is called the focal element of the evidence in the belief functions theory, if $m(A)>0$. Let $\mathcal{A}$ be the set of all focal elements of the evidence. Then a pair $F=(\mathcal{A}, m)$ is called a body of evidence. Evaluating the conflict between multiple evidence (external conflict) given on the set $X$, as well as evaluating the internal conflict of each evidence are important questions of the evidence theory. The conjunctive conflict measure is historically the first and most popular measure of external conflict. This measure is calculated by the formula

$$
K=K\left(F_{1}, F_{2}\right)=\sum_{A \cap B=\emptyset} m_{1}(A) m_{2}(B)
$$

for two bodies of evidence $F_{1}=\left(\mathcal{A}_{1}, m_{1}\right)$ and $F_{2}=$ $\left(\mathcal{A}_{2}, m_{2}\right)$ on $X$. It is not difficult to see that $K \in$ $[0,1]$ and the value $K=1$ corresponds to the absolute conflict of the two bodies of evidence: $A \cap B=\emptyset$ for all $A \in \mathcal{A}_{1} \quad B \in \mathcal{A}_{2}$. We will use this measure below to assess the degree of conflicting political party positions. The results of studies of the axiomatics, properties and approaches of the defining of external conflict measures can be found in $[3,8,18,20,21]$.

Internal conflict of evidence characterizes the degree of its heterogeneity. In particular, if the evidence contains non-intersecting focal elements with large masses, then this indicates about a great internal conflict of such evidence. There are various ways to evaluate internal conflict $[6,17,24]$. For example, the value $K(F, F)$ (so-called autoconflict) can be considered a measure of the internal conflict of the body of evidence of $F=(\mathcal{A}, m)$. Here the measure $K$ is calculated by 
the formula (1). In [6] a measure of internal conflict was introduced using the formula

$$
K_{\text {int }}(F)=1-\max _{x} P l(x)=\min _{x} \operatorname{Bel}(X \backslash\{x\}) .
$$

We will use this value to estimate the internal conflict of evidence.

\section{Setting the Main Objectives of the Study}

Let there be $r$ parties, which received $p_{1}, \ldots, p_{r}$ votes in the elections, respectively. In addition, there is a list of $n$ issues $N_{n}=\{1, \ldots, n\}$ for which all parties have voiced their positions before the elections; let $2^{N_{n}}$ be the set of all subsets of the list of issues. The party can answer each of the questions either 'yes' or 'no'. Therefore, for any set questions $A \in 2^{N_{n}}$, there are $2^{|A|}$ subsets, taking into account different answers to questions from the $A$. Let $2^{A}$ are the set of all such answers to the questions from $A$. Then $2^{\overline{N_{n}}}=\bigcup_{A \in 2^{N_{n}}} 2^{A}$ is the set of all the subsets of the list of questions with the various answers, $\left|2^{\overline{N_{n}}}\right|=3^{n}$. Each set $A \in 2^{N_{n}}$ can be encoded by the $a=\left(a_{1}, \ldots, a_{n}\right), a_{i}=1$ if $x_{i} \in A$ and $a_{i}=0$ otherwise. Each set $A \in 2^{\overline{N_{n}}}$ can be encoded by the $a=\left(a_{1}, \ldots, a_{n} ; a_{n+1}, \ldots, a_{2 n}\right)$ : if $x_{i} \in A$ and the answer was 'yes', then $a_{i}=1$; if $x_{i} \in A$ and the answer was 'no', then $a_{n+i}=1$ and $a_{i}=0$ otherwise.

Example 1. Let $n=2$. Then the vector $(0,0 ; 0,0)$ corresponds to the empty set of questions. Two vectors $(1,0 ; 0,0)$ and $(0,0 ; 1,0)$ correspond to the set $A=\{1\}$ (the $1^{\text {st }}$ question and two possible answers 'yes' or 'no'). Two vectors $(0,1 ; 0,0)$ and $(0,0 ; 0,1)$ correspond to the set $A=\{2\}$ (the $2^{\text {nd }}$ question and two possible answers 'yes' or 'no'). Four vectors $(1,1 ; 0,0)-$ 'yes-yes', (1,0;0,1) - 'yes-no', ( 0,$1 ; 1,0)$ - 'no-yes', $(0,0 ; 1,1)$ - 'no-no' correspond to the set of questions $A=\{1,2\}$. We have $\left|2^{\overline{N_{2}}}\right|=3^{2}=9$ vectors from $2^{\overline{N_{2}}}$.

\subsection{Finding the Most Significant Groups of Issues for Voting and Assessment of Political Heterogeneity of the Society}

It is necessary to determine the significance of a particular subset of issues for voting, i.e. need to find the mass function $m: 2^{N_{n}} \rightarrow[0,1]$, where the values $m(A)$ characterize the shares of voters who consider the questions of the set $A$ as significant, $\sum_{A} m(A)=1$. Instead of the set function $m$, it is easier to find the set function $\bar{m}: 2^{\overline{N_{n}}} \rightarrow[0,1]$. Then $\sum_{B \in 2^{A}} \bar{m}(B)=m(A)$, $A \in 2^{N_{n}}$.

Let $v_{k, i}=\left\{\begin{aligned} & 1, \text { if } i \in\{1, \ldots, n\} \text { and } k \text {-th party answered } \\ & \text { 'yes' to the } \mathrm{i}-\text { th question, } \\ & 1, \text { if } i \in\{n+1, \ldots, 2 n\} \text { and } k \text {-th party } \\ & \text { answered 'no' to the }(\mathrm{i}-\mathrm{n}) \text {-th question, } \\ & 0, \text { otherwise, }\end{aligned}\right.$

$k=1, \ldots, r, \quad i=1, \ldots, 2 n$, and $V_{k}=$ $\left(v_{k, 1}, \ldots, v_{k, n} ; v_{k, n+1}, \ldots, v_{k, 2 n}\right)$ be the profile of answers to questions of the $k$-th party. It is easy to see that equality $v_{k, s}+v_{k, n+s}=1$ is true for the profile of the $k$-th party for all $s=1, \ldots, n$. For example, for $n=4$ questions, the profile $V=(1,0,0,1 ; 0,1,1,0)$ means that the party answered 'yes' to the $1^{\text {st }}$ and $4^{\text {th }}$ questions and 'no' to the $2^{\text {nd }}$ and $3^{\text {rd }}$ questions.

We have $\min _{i \in A}\left\{v_{k, i}\right\}=1$ if the set of answers $A$ is contained in the profile of the $k$-th political party, and we have $\min _{i \in A}\left\{v_{k, i}\right\}=0$ otherwise. Then the value

$$
C\left(V_{k}\right)=\sum_{A \in 2^{\overline{N_{n}}}} \bar{m}(A) \min _{i \in A}\left\{v_{k, i}\right\}
$$

is proportional to the potential number of voters of the $k$-th party. It is necessary to find such values $\{m(A)\}$ in order to minimize the discrepancy between the distributions $\left(p_{k}\right)$ and $\left(C\left(V_{k}\right)\right)$ (after normalization). In particular, we can estimate the degree of proportionality of the values in the bits: $\frac{C\left(V_{k}\right)}{C\left(V_{j}\right)} \approx \frac{p_{k}}{p_{j}}$ for any pairs of indices $k, j \in\{1, \ldots, r\}$. We will consider the functional

$$
R=\sum_{i=1}^{r}\left(p_{i+1} C\left(V_{i}\right)-p_{i} C\left(V_{i+1}\right)\right)^{2}
$$

$\left(p_{r+1}=p_{1}, V_{r+1}=V_{1}\right)$ as a criterion for minimizing. Thus, it is necessary to find the values $\left\{\bar{m}(A): A \in 2^{\overline{N_{n}}}\right\}$, satisfying the conditions

$$
\sum_{A \in 2^{N_{n}}} \bar{m}(A)=1, \quad \bar{m}(A) \geq 0 \quad \forall A \in 2^{\overline{N_{n}}}
$$

and minimizing the criterion $R$. This is a quadratic programming problem. Expression (2) is the Choquet integral of the function $V_{k}=\left(v_{k, 1}, \ldots, v_{k, 2 n}\right)$ with respect to the belief measure $\operatorname{Bel}(A)=\sum_{B \subseteq A} \bar{m}(B)$.

Example 2. Suppose that three parties $A, B$ and $C$ participated in the election. These parties indicated their position on four issues before the election. The responses of the parties to these four questions are presented in Table 1. These parties received 50\%, 40\% and $10 \%$ of the vote respectively.

Which groups of questions should be considered more significant? Suppose that only a pairs of questions interest us. Then those pairs of questions will be more 


\begin{tabular}{|c||c|c|c|}
\hline & $A$ & $B$ & $C$ \\
\hline 1 & 1 & 1 & 0 \\
2 & 0 & 1 & 1 \\
3 & 1 & 0 & 0 \\
4 & 0 & 0 & 0 \\
\hline votes & $50 \%$ & $40 \%$ & $10 \%$ \\
\hline
\end{tabular}

Table 1: Party responses and vote distribution.

significant, to which the $A$ and $B$ parties, which received the largest number of votes, answered about the same, and the answers of the $C$ party, on the contrary, were very different from those of the $A$ and $B$ parties. It is easy to see that the smallest discrepancies in the answers of the parties $A$ and $B$ were on pairs of questions $\{1,4\}$ (0 discrepancies), $\{1,2\}$, $\{1,3\},\{2,4\},\{3,4\}$ (1 discrepancies each). But the greatest discrepancy between the responses of the parties $A, B$ on the one hand and the $C$ party on the other hand to these five pairs of questions will be in the pairs of questions $\{1,2\}$ and $\{1,3\}$ (3 discrepancies each). These pairs should be considered the most significant.

Let $\left\{A_{j}\right\}_{j=1}^{3^{n}-1}$ is some ordering of all subsets of $2^{\overline{N_{n}}}$, $\mathbf{m}=\left(m_{j}\right), m_{j}=\bar{m}\left(A_{j}\right), c_{k, j}=\min _{i \in A_{j}}\left\{v_{k, i}\right\}, j=$ $1, \ldots, 3^{n}-1, k=1, \ldots, r, \mathbf{c}_{k}$ is the $k$-th row vector of the matrix $\mathbf{C}=\left(c_{k j}\right)$. Then $C\left(V_{k}\right)=\mathbf{c}_{k} \mathbf{m}$ and the following statement is true.

Proposition 1. The solution of the problem of minimizing the functional $R$ under conditions (3) is equivalent to solving the problem

$$
\left\{\begin{array}{l}
\mathbf{m}^{T} \mathbf{Q m} \rightarrow \min \\
m_{j} \geq 0 \forall j \\
\sum_{j} m_{j}=1
\end{array}\right.
$$

where

$$
\mathbf{Q}=\sum_{k=1}^{r-1}\left(p_{k+1} \mathbf{c}_{k}-p_{k} \mathbf{c}_{k+1}\right)^{T}\left(p_{k+1} \mathbf{c}_{k}-p_{k} \mathbf{c}_{k+1}\right)
$$

Note that if $\min _{k} \min _{i \in A}\left\{v_{k, i}\right\}=0$ is true for some subset $A \in 2^{\overline{N_{n}}}$, then the variable corresponding to this set $A$ can be excluded from the vector $\mathbf{m}$. Therefore, the real dimension of the vector $\mathbf{m}$ is much less than $3^{n}$.

Since $\operatorname{rank} \mathbf{Q} \leq r \ll 3^{n}$, then problem (4) can have many solutions. To solve such an ill-posed problem, instead of the functional $\mathbf{m}^{T} \mathbf{Q m}$ in (4), we can consider its regularization $\mathbf{m}^{T} \mathbf{Q} \mathbf{m}-\alpha\|\mathbf{m}\|^{2}=\mathbf{m}^{T}(\mathbf{Q}-\alpha) \mathbf{m}$, where $\alpha>0$ is a regularization parameter. Regularization more 'fines' those distributions $\mathbf{m}$ that are closer to a uniform distribution.
Note that the minimum value of the criterion $R$ can be zero if the system

$$
p_{k+1} C\left(V_{k}\right)=p_{k} C\left(V_{k+1}\right), \quad k=1, \ldots, r
$$

has a solution. From (2)) it follows that this system is equivalent to a linear system

$$
\begin{gathered}
\sum_{A \in 2^{N_{n}}} \bar{m}(A) b_{k}(A)=0, \quad k=1, \ldots, r, \\
\sum_{A \in 2^{\overline{N_{n}}}} \bar{m}(A)=1, \quad \bar{m}(A) \geq 0 \quad \forall A \in 2^{\overline{N_{n}}},
\end{gathered}
$$

where $b_{k}(A)=p_{k+1} \min _{i \in A}\left\{v_{k, i}\right\}-p_{k} \min _{i \in A}\left\{v_{k+1, i}\right\}$, $k=1, \ldots, r, A \in 2^{\overline{N_{n}}}$.

After finding the mass function $m: 2^{N_{n}} \rightarrow[0,1]$, we find the belief function $\operatorname{Bel}(A)=\sum_{B \subset A} m(B)$, which characterizes the degree of belief in the fact that the set of questions $A$ is significant for voting. The contribution of individual issues to the overall significance is estimated using Shapley values.

The political heterogeneity of society will be assessed using a measure of internal conflict of the body of evidence $F=(\mathcal{A}, m)$ determined on the set $N_{n}$ of all issues. If completely different non-intersecting (or 'slightly intersecting') groups of electoral issues are significant, then this means greater heterogeneity of society. We will use the value $K_{\text {int }}(F)=$ $\min _{i} \operatorname{Bel}\left(N_{n} \backslash\{i\}\right)$ as a measure of internal conflict. $K_{\text {int }}(F)=\min _{i} \operatorname{Bel}\left(N_{n} \backslash\{i\}\right)$ (see Section 2).

\subsection{Evaluation of the Influence of Party Positions on Voting}

Consider the projection of evidence of the significance of questions for the results of voting on the profiles of separate parties.

We will say that a subset $A \in 2^{\overline{N_{n}}}$ is contained in the profile of the $k$-th party $V_{k}$ (and denote it $A \subseteq V_{k}$ ) if $\forall i \in A: v_{k, i}=1$. Let $\mathcal{A}$ be the set of all the focal elements found in the previous problem, $\mathcal{A}_{k}=\{A \in$ $\left.\mathcal{A}: A \subseteq V_{k}\right\}$ is the projection of the focal elements on the profile of the $k$-th party and $\bar{m}_{k}(A)=\bar{m}(A)$, if $A \in$ $\mathcal{A}_{k}$. The value of $\bar{m}_{k}(A)$ characterizes the electoral significance for the $k$-th party position on the set of issues $A \in \mathcal{A}_{k}$. Then the value $M_{k}=\sum_{A \in \mathcal{A}_{k}} \bar{m}_{k}(A)$ characterizes the degree of significance of the position of the $k$-th party on the result of voting for it. It is not difficult to see that $M_{k} \in[0,1]$, and if $M_{k}=0$, then the party position on all issues is insignificant for the elections. If $M_{k}=1$, then the political party positions on all issues is significant for winning the election. 


\subsection{Estimates of Inconsistency of Party Positions on Significant Issues}

The analysis of the proximity of political party positions on key issues is essential in the case of decisions on the formation of coalitions or other joint actions. This can be done within the framework of the belief functions theory, if we analyze the conflict between the evidence of the electoral significance of the political party positions on a set of issues.

For this purpose, we will form new bodies of evidence $\left\{F_{k}\right\}$ on the set functions $\left\{\bar{m}_{k}\right\}: F_{k}=\left(\mathcal{A}_{k}, m_{k}^{\prime}\right), m_{k}^{\prime}=$ $\bar{m}_{k} / M_{k}$, if $M_{k} \neq 0$. If $M_{k}=0$, then this indicates about insignificance of the political party position on the election result. Therefore, in this case, the position of the $k$-th party will be non-conflict with the positions of other parties in the considered sense (although, of course, the political party positions may be conflicting with respect to other measures).

We will use the measure of external conflict (1) to assess the degree of conflict in the political party positions. The value $K_{i, j}=K\left(F_{i}, F_{j}\right), K_{i, j} \in[0,1]$ characterizes the degree of conflict (inconsistency) between the positions of the $i$-th and $j$-th parties. The value $K_{i, j}=1$ means that the positions of the $i$-th and $j$-th parties do not overlap on all issues and their various combinations important for voting. The value $K_{i, j}=0$ means that the $i$-th and $j$-th parties have common positions on all combinations of issues that are significant for voting.

\section{Data Description}

In this article, we used data obtained from the site The German Federal Agency for Civic Education [31] on the positions of 28 parties (or blocs) of Germany on topical issues before the 2013 Bundestag elections. There were 38 questions in the list. Each party (or block) should have answered 'yes' or 'no' to each question. In addition, the results of the 2013 Bundestag election were used in the study [30].

We preliminarily selected 8 questions for which the population gave the largest number of votes to reduce the computational complexity and visibility of the results. There are, for example, issues such as: \#22 on raising the retirement age (3 490000 people voted for inclusion of this issue in the list), \#18 on the state student financing system (2 060000 people voted for inclusion of this issue in the list), \#2 on subsidizing families with children who do not attend school (1 010000 people voted for inclusion of this issue in the list), etc.

In addition, 6 parties (or blocks) that gained more than $3 \%$ of votes in parliamentary elections were selected for further research. These are the block of the Christian Democratic Union of Germany and the Christian Social Union in Bavaria (CDU/CSU), Social Democratic Party of Germany (SPD), The Left (DIE LINKE), Alliance 90/The Greens (GRUNE), Free Democratic Party (FDP), Alternative for Germany (AfD) [30].

\section{$5 \quad$ Research Results}

Results of Finding the Most Significant Groups of Questions for Voting. Analysis of the response profiles revealed 1003 subsets $A$ of questions for a set of 8 questions and 6 parties (or blocks) for which $\min _{k} \min _{i \in A}\left\{v_{k, i}\right\}>0$. This is the real dimension of the vector $\mathbf{m}$.

The 255 subsets of questions with non-zero values of the mass function (focal elements) were found in the analysis of the most significant for voting subsets of 8 questions by the method described in subsection 3.1. The group of the most significant subsets consists of 30 sets of questions with a significance of 0.0072. Questions \#2, \#18 and \#22 are included in all subsets with the greatest significance. The significance of these particular questions is confirmed by the Shapley vector. The Shapley values will be equal to $0.18,0.15,0.12$ for questions \#2, \#8, \#22, respectively, and approximately 0.11 for the remaining 5 questions.

Evaluating the Political Heterogeneity of Society Using a Measure of Internal Conflict of Evidence of the Importance of Issues. This measure (see Subsection 3.1) in our case will be equal to $K_{\text {int }}(F)=0.264$. This indicates a certain political homogeneity of society (although, of course, this measure is relative).

The Results of the Assessment of the Significance of the Political Party Positions of Germany for Voting, calculated using the formula $M_{k}=$ $\sum_{A \in \mathcal{A}_{k}} \bar{m}_{k}(A), k=1, \ldots, r$ (see Section 3.2), are presented in the graph in Figure 1. There is also a graph of the shares of votes in elections for each of the 6 parties. It can be seen that the graphs of significance and the percentage of votes in elections strongly correlate with each other.

The Results of the Assessment of the Conflicts of the Party Positions on Significant Issues, calculated using the conflict measure (see Subsection 3.3), are presented in Figure 2.

Note that the CDU/CSU block has a large measure of conflict with the parties SPD, DieLinke and GRUNE, which were the closest pursuers of the block in the elections. On the other hand, the positions of the $\mathrm{CDU} / \mathrm{CSU}$ block coincide in the main sets of questions relevant for voting with the positions of the FDP and 


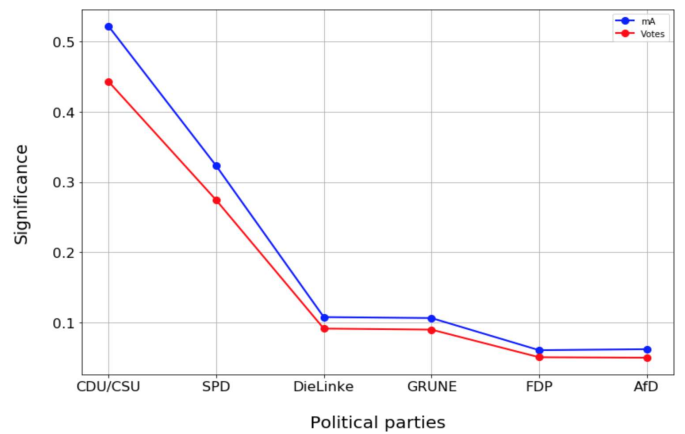

Figure 1: Significance of party positions on a set of 8 questions for voting results

\begin{tabular}{c|c|c|c|c|c|c}
\hline & CDU/CSU & SPD & Dielinke & GRUNE & FDP & AfD \\
\hline CDU/CSU & & 0,35 & 0,59 & 0,42 & 0,14 & 0,14 \\
\hline SPD & & & 0,12 & 0,16 & 0,40 & 0,20 \\
\hline Dielinke & & & & 0,13 & 0,32 & 0,48 \\
\hline GRUNE & & & & & 0,20 & 0,36 \\
\hline FDP & & & & & & 0,18 \\
\hline AfD & & & & & & \\
\hline
\end{tabular}

Figure 2: Conflict of the party positions on significant issues: a red color corresponds to a greater conflict of positions; a greener color corresponds to a smaller conflict (greater consistency of positions)

AfD parties. The opposition parties SPD, DieLinke and GRUNE have similar political views, and this is reflected in their meanings of pairwise conflict measures.

In the previous elections to the Bundestag in 2009, a coalition of the parties CDU/CSU and FDP was formed. The model confirms the consistency of the positions of these parties. The AfD and CDU / CSU parties are right-wing parties, and this also implies the possibility of consistency in their positions. If we compare the obtained results with real coalitions, then we can conclude that the considered model is correct.

\section{Conclusion}

In this paper, the methods of the theory of nonadditive measures (including integration by such measures), as well as the methods of the belief functions theory (including evaluation of internal and external conflict of evidence) are applied to solving a number of problems of analyzing the significance and conflict of the political party positions. The study was conducted on a database of online polls of parties in Germany before the elections to the Bundestag in 2013 and the results of these elections.

In particular, the possibility of solving the following problems using the methods of the theory of nonadditive measures and the belief functions theory is shown:
- the finding the most significant groups of questions for voting by minimizing the discrepancy between the distribution of votes among the parties in elections and the prior distribution of potential votes among the parties, calculated using the Choquet integral according to online polls of parties before the elections; we note that the construction of the Choquet integral appears naturally when trying to estimate the number of those who are ready to vote for a given political party;

- evaluation of the political heterogeneity of society through a measure of internal conflict evidence of the importance of issues;

- evaluation of the significance of the positions of individual parties for voting in elections by building projections of evidence of the importance of questions on the profiles of individual parties;

- evaluation of conflict of political party positions on significant issues using a measure of external conflict.

\section{Acknowledgement}

The authors are grateful to Prof. Andranik Tangian (WSI in the Hans-Böckler-Foundation and Karlsruhe Institute of Technology, Düsseldorf, Germany) for initiating this study and for the data provided. The financial support from the Government of the Russian Federation within the framework of the implementation of the 5-100 Programme Roadmap of the National Research University Higher School of Economics is acknowledged. This work was also partially supported by the grant 18-01-00877 of RFBR (Russian Foundation for Basic Research).

\section{References}

[1] F. Aleskerov, H. Ersel, Y. Sabuncu, Power and coalitional stability in the Turkish Parliament (1991-1999), Turkish Studies 1(2) (2000) 21-38.

[2] J. Bernauer, T. Brauninger, Intra-party preference heterogeneity and faction membership in the 15th German Bundestag: a computational text analysis of parliamentary speeches, German Politics 18(3) (2008) 385-402.

[3] A. Bronevich, A. Lepskiy, H. Penikas, The application of conflict measure to estimating incoherence of analyst's forecasts about the cost of shares of Russian companies, Procedia Computer Science 55 (2015) 1113-1122.

[4] A. Chateauneuf, J.-Y. Jaffray, Some characterizations of lower probabilities and other monotone 
capacities through the use of Möbius inversion, Mathematical Social Sciences 17 (1989) 263-283.

[5] G. Choquet, Theory of capacities, Annales de l'Institut Fourier 5 (1953) 131-295.

[6] M. Daniel, Conflicts within and between belief functions, in: E. H. Hüllermeier, et al. (Eds.), IPMU 2010, LNAI, Vol. 6178, Springer, Heidelberg, 2010, pp. 696-705.

[7] A. P. Dempster, Upper and lower probabilities induced by multivalued mapping, Ann. Math. Statist. 38 (1967) 325-339.

[8] S. Destercke, T. Burger, Toward an axiomatic definition of conflict between belief functions, IEEE Transactions on Cybernetics 43(2) (2013) 585596.

[9] X. Du, Multiple instance Choquet integral for multiresolution sensor fusion, Ph.D thesis, University of Missouri, Columbia, MO (2017).

[10] M. Grabisch, Fuzzy integral for classification and feature extraction, in: M. Grabisch, T. Murofushi, M. Sugeno (Eds.), Fuzzy Measures and Integrals: Theory and Applications, Physica Verlag, Heidelberg, 2000, pp. 415-434.

[11] M. Grabisch, Set Functions, Games and Capacities in Decision Making, Springer, Cham, 2016.

[12] M. Grabisch, J.-M. Nicolas, Classification by fuzzy integral: performance and tests, Fuzzy Sets and Systems 65(2-3) (1994) 255-271.

[13] M. Grabisch, M. Roubens, Application of the Choquet integral in multicriteria decision making, in: M. Grabisch, T. Murofushi, M. Sugeno (Eds.), Fuzzy Measures and Integrals: Theory and Applications, Physica Verlag, Heidelberg, 2000, pp. 348-374.

[14] R. Harmel, H. Uk, A. Tan, K. Janda, Performance, leadership, factions, and party change: an empirical analysis, West European Politics 18(1) (1995) 1-33.

[15] J. M. Keller, P. D. Gader, H. Tahani, J.-H. Chiang, M. Mohamed, Advances in fuzzy integration for pattern recognition, Fuzzy Sets and Systems 65 (1994) 273-283.

[16] D. Landa, Meirowitz A. Game theory, information, and deliberative democracy, American Journal of Political Science 53(2) (2009) 427-444.

[17] A. Lepskiy, On internal conflict as an external conflict of a decomposition of evidence, in: J. Vejnarová, V. Kratochvil (Eds.), Belief Functions:
Theory and Applications, LNAI 9861, SpringerVerlag, 2016, pp.25-34.

[18] A. Lepskiy, On the conflict measures agreed with the combining rules, in: S. Destercke, S. Denoeux, F. Cuzzolin, A. Martin (Eds.), Belief Functions: Theory and Applications, LNCS 11069, SpringerVerlag, Cham, 2018, pp.172-180.

[19] G. Levy, A Model of Political Parties, Journal of Economic Theory 115(2) (2004) 250-277.

[20] W. Liu, Analysing the degree of conflict among belief functions, Artificial Intelligence 170 (2006) 909-924.

[21] A. Martin, About conflict in the theory of belief functions, in: Belief Functions: Theory and Applications, AISC 164, Springer-Verlag, 2012, pp. $161-168$.

[22] N. Persico, J. Rodriguez-Pueblita, D. Silverman, Factions and political competition, Journal of Political Economy 2 (2011) 242-288.

[23] N. Schofield, Stability of coalition governments in Western Europe: 1945-1986, European Journal of Political Economy 3(4) (1987) 555-591.

[24] J. Schubert, The internal conflict of a belief function, in: T. Denoeux, M-H. Masson (Eds.) Belief Functions: Theory and Applications, AISC 164, Springer-Verlag, 2012, pp. 169-177.

[25] G. Shafer, A Mathematical Theory of Evidence, Princeton University Press, Princeton, N.J., 1976.

[26] L. Shapley, A value for n-person games, Annals of Mathematics Studies 28 (1953) 307-317.

[27] P. Smets, Decision making in TBM: the necessity of the pignistic transformation, International Journal of Approximate Reasoning 38 (2005) 133147.

[28] K.-L. Su, Y.-M. Jau, J.-T. Jeng, Modeling of nonlinear aggregation for information fusion systems with outliers based on the Choquet integral, Sensors 11 (2011) 2426-2446.

[29] A. Tangian, Mathematical Theory of Democracy, Springer-Verlag, Berlin Heidelberg, 2014.

[30] The German Bundestag: http://www.bundestag.de/ (date of access: 18.01.2019).

[31] The German Federal Agency for Civic Education: http://www.bpb.de/politik/ (date of access: 18.01.2019) 